\title{
Double cancer comprising malignant pleural mesothelioma and squamous cell carcinoma of the lung treated with radiotherapy: A case report
}

\author{
YOSHIKI NEGI ${ }^{1}$, KOZO KURIBAYASHI ${ }^{1}$, HIROSHI DOI ${ }^{2}$, NORIHIKO FUNAGUCHI ${ }^{3}$, YUICHI KODA ${ }^{1}$, \\ ERIKO FUJIMOTO $^{1}$, KOJI MIKAMI ${ }^{1}$, TOSHIYUKI MINAMI ${ }^{1}$, TAKASHI YOKOI ${ }^{1,4}$ and TAKASHI KIJIMA ${ }^{1,4}$ \\ ${ }^{1}$ Division of Respiratory Medicine, Department of Internal Medicine and ${ }^{2}$ Department of Radiology, \\ Hyogo College of Medicine, Nishinomiya, Hyogo 663-8501; ${ }^{3}$ Department of Respiratory Medicine, \\ Murakami Memorial Hospital, Asahi University, Mizuho, Gifu 500-8523; ${ }^{4}$ Department of Thoracic Oncology, \\ Hyogo College of Medicine, Nishinomiya, Hyogo 663-8501, Japan
}

Received January 10, 2018; Accepted June 8, 2018

DOI: $10.3892 / \mathrm{mco} .2018 .1650$

\begin{abstract}
Pleurectomy/decortication (P/D) is the surgical treatment of choice for early malignant mesothelioma, but it remains unclear whether radiotherapy along with $\mathrm{P} / \mathrm{D}$ should be used as multimodal treatment for this disease. We herein present the case of a 76-year-old man with a history of asbestos exposure who was diagnosed with left-sided malignant pleural mesothelioma in February 2010. The patient underwent chemotherapy with a combination of cisplatin and pemetrexed and achieved stable disease, after which time he was kept under observation. A positron emission tomography/computed tomography scan performed in February 2011 revealed nodular shadows with fluorodeoxyglucose uptake in S3 of the left lung; using bronchoscopy, the patient was diagnosed with stage IIB (cT3NOM0) primary squamous cell carcinoma. Chemoradiotherapy with vinorelbine and 60 Gy/20 fr radiotherapy was performed, and a partial response was obtained, suggesting that the radiotherapy used to treat the carcinoma of the lung may have also helped control the disease activity of the pre-existing mesothelioma. The present case indicates the value of radiotherapy in the treatment of malignant mesothelioma. The aim of the present study was to examine the
\end{abstract}

Correspondence to: Dr Kozo Kuribayashi, Division of Respiratory Medicine, Department of Internal Medicine, Hyogo College of Medicine, 1-1 Mukogawa-cho, Nishinomiya, Hyogo 663-8501, Japan E-mail: kuririn@hyo-med.ac.jp

Abbreviations: BED, biologically effective dose; CT, computed tomography; EPP, extrapleural pneumonectomy; FDG, fluorodeoxyglucose; IMRT, intensity-modulated radiotherapy; P/D, pleurectomy/decortication; PET, positron emission tomography

Key words: malignant pleural mesothelioma, squamous cell carcinoma, radiotherapy, trimodal treatment, intensity-modulated radiotherapy, pleurectomy/decortication possibility of new multimodal treatments for mesothelioma, along with a discussion of the relevant literature.

\section{Introduction}

Exposure to asbestos is known to increase the incidence of mesothelioma as well as that of lung cancer; however, reports of cases of double cancers comprising these two cancer types are rare (1).

The efficacy of radiotherapy for multimodal treatment of mesothelioma and lung cancer has been reported previously (2). However, only few reports have described the use of radiotherapy for two different tumors in the same patient.

In the present study, we report the case of a patient with double cancer comprising malignant pleural mesothelioma and squamous cell lung cancer, in whom radiotherapy used to treat the lung cancer may have helped control the progression of the malignant pleural mesothelioma. This case is reported together with a discussion of the literature, as it provides valuable insight into the future positioning of radiotherapy in multimodal therapy for malignant pleural mesothelioma.

\section{Case report}

The patient was a 75-year-old man with a history of early gastric cancer who was employed at a cement factory, with a history of exposure to asbestos from the age of 25 to 40 years.

The patient was first seen at the asbestos center of our hospital in November 2005 for a Hyogo Labor Bureau Asbestos Examination in accordance with the patient's Asbestos Health Monitoring Handbook; thereafter, he underwent periodic examinations for pulmonary asbestosis and bilateral pleural thickening.

In October 2009, chest radiography (Fig. 1A) revealed left pleural effusion that had not been present on the previous (March 2009) chest radiography images (Fig. 1B). However, chest computed tomography (CT) revealed no worsening of the pleural lesions; thus, a strategy of follow-up observation was selected (Fig. 2). Approximately 3 months later, the patient 
developed exertional dyspnea, and the left pleural effusion worsened over time; therefore, the pleural effusion was aspirated and subjected to cytological examination. Cytological examination detected class $\mathrm{V}$ malignant mesothelioma (Fig. 3A). In February 2010, the patient underwent a pleural surgical biopsy under general anesthesia and was diagnosed with malignant pleural mesothelioma, International Mesothelioma Interest Group classification cTlaN0M0, stage 1A (Fig. 3B). The patient was not a candidate for extrapleural pneumonectomy (EPP) due to the impaired pulmonary function caused by pulmonary asbestosis and chronic obstructive pulmonary disease; therefore, a strategy of chemotherapy alone was selected, and the patient was administered cisplatin $\left(75 \mathrm{mg} / \mathrm{m}^{2}\right.$ on day 1 then every 21 days) plus pemetrexed $\left(500 \mathrm{mg} / \mathrm{m}^{2}\right.$ on day 1 then every 21 days) from April 2010 onwards. After completing four courses of chemotherapy, the left pleural effusion improved and stable disease was achieved.

In February 2011, fluorodeoxyglucose (FDG) positron emission tomography (PET)/CT revealed the emergence of $20-\mathrm{mm}$ nodular shadows with irregular margins in two locations, with FDG uptake in the S3 of the left lung (Fig. 4).

The patient was admitted to our department for detailed examination and treatment. Upon admission, right-sided hemiparesis caused by the after-effects of a left cerebral infarction was observed; the superficial lymph nodes were not palpable. On auscultation, a fine crackle was heard in the posterior portion of both lower lung fields. There was no digital clubbing, and the $\mathrm{SpO}_{2}$ was $95 \%$ (room air).

The laboratory findings upon admission are listed in Table I. All blood cell counts and biochemical tests were normal. The KL-6 levels were mildly elevated to $638 \mathrm{U} / \mathrm{ml}$ (normal, $<500 \mathrm{U} / \mathrm{ml}$ ). The tumor markers carcinoembryonic antigen, squamous cell carcinoma (SCC) antigen, cytokeratin-19 fragments and pro-gastrin-releasing peptide were all within the normal ranges.

Chest radiography revealed two $15-\mathrm{mm}$ nodular shadows with irregular margins in the left central lung field. The images showed circumferential pleural thickening and partial coverage by pleural plaques. Chest contrast-enhanced CT revealed a reticulate shadow in both lung fields; scattered nodular shadows with a centrilobular distribution were observed. Two 20-mm nodular shadows with irregular margins were detected in $\mathrm{S} 3$ of the left lung (Fig. 5A). Pleural plaques and pleural thickening were observed bilaterally, along with thickening of the left interlobular pleura. Contrast enhancement was evident, and exacerbation of the mesothelioma lesions was suspected (Fig. 5B).

Following admission, the left S3 nodular shadows were examined by bronchoscopy, and a diagnosis of SCC (cT3N0M0 stage IIA) was confirmed (Fig. 6). Thus, the patient was diagnosed with a double cancer comprising malignant pleural mesothelioma and SCC. He was not considered to be a candidate for surgery due to the impaired pulmonary function; therefore, chemoradiotherapy with vinorelbine $\left(10 \mathrm{mg} / \mathrm{m}^{2}\right.$ on day 1 then every 7 days) and radiotherapy (60 Gy in 20 fractions at 3 Gy per fraction) were administered. The patient was discharged without complications on day 59 after admission. Although the patient later developed a treatment-related complication (grade II radiation pneumonitis), the primary tumor was well-controlled.
Table I. Laboratory findings upon admission.

\begin{tabular}{lcc}
\hline Tests & Values & $\mathrm{Units}$ \\
\hline Total protein & 6.9 & $\mathrm{~g} / \mathrm{dl}$ \\
Albumin & 4.0 & $\mathrm{~g} / \mathrm{dl}$ \\
Total bilirubin & 0.4 & $\mathrm{mg} / \mathrm{dl}$ \\
AST & 24 & $\mathrm{U} / 1$ \\
ALT & 37 & $\mathrm{U} / 1$ \\
LDH & 163 & $\mathrm{U} / 1$ \\
ALP & 321 & $\mathrm{U} / 1$ \\
$\gamma$ GTP & 46 & $\mathrm{U} / 1$ \\
BUN & 10 & $\mathrm{mg} / \mathrm{dl}$ \\
Creatinine & 0.64 & $\mathrm{mg} / \mathrm{dl}$ \\
Na & 139 & $\mathrm{mmol} / 1$ \\
K & 3.6 & $\mathrm{mmol} / 1$ \\
Cl & 101 & $\mathrm{mmol} / 1$ \\
Ca & 8.9 & $\mathrm{mg} / \mathrm{dl}$ \\
CRP & 5.8 & $\mathrm{mg} / \mathrm{dl}$ \\
WBC count & 5,910 & $/ \mu 1$ \\
RBC count & 476 & $\mathrm{x} 10^{4} / \mu \mathrm{g}$ \\
Hb & 13.4 & $\mathrm{~g} / \mathrm{dl}$ \\
HCT & 37.9 & $\%$ \\
MCV & 79.6 & $\mathrm{fl}$ \\
MCHC & 35.4 & $\mathrm{pg}$ \\
PLT count & 27.1 & $\mathrm{x} 10^{4} / \mu \mathrm{g}$ \\
KL-6 & 638 & $\mathrm{U} / \mathrm{ml}$ \\
CEA & 1.4 & $\mathrm{ng} / \mathrm{ml}$ \\
SCC & 1.1 & $\mathrm{ng} / \mathrm{ml}$ \\
CYFRA & $<1.0$ & $\mathrm{ng} / \mathrm{ml}$ \\
ProGRP & 31.8 & $\mathrm{pg} / \mathrm{ml}$ \\
\hline ALP, & & \\
\hline
\end{tabular}

ALP, alkaline phosphatase; ALT, alanine transaminase; AST, aspartate aminotransferase; BUN, blood urea nitrogen; CEA, carcinoembryonic antigen; CRP, C-reactive protein; CYFRA, cytokeratin-19 fragments; Hb, hemoglobin; HCT, hematocrit; LDH, lactate dehydrogenase; MCHC, mean corpuscular hemoglobin concentration; MCV, mean corpuscular volume; PLT, platelet; Pro-GRP, pro-gastrin-releasing peptide; RBC, red blood cell; SCC, squamous cell carcinoma; WBC, white blood cell.

In September 2011, FDG PET/CT revealed the emergence of a new 20-mm nodular shadow with irregular margins and FDG uptake in the left S8. Since the left S3 primary tumor was well-controlled, this was not considered a recurrence, but a second primary cancer was suspected. On November 2011, bronchoscopy was performed, and the diagnosis of SCC (cT1bNOM0 stage IA) was confirmed.

As the patient still suffered from grade II radiation pneumonitis, radiotherapy for the S8 lesion was deemed high-risk, and it was decided that chemotherapy would be implemented after the radiation pneumonitis had subsided.

While waiting for the radiation pneumonitis to subside, in March 2012, docetaxel chemotherapy was started $\left(60 \mathrm{mg} / \mathrm{m}^{2}\right.$ on day 1 then every 21 days), but the disease activity of the S8 lesion was worse after four courses. In October 2012, chemotherapy was changed to four courses of gemcitabine 

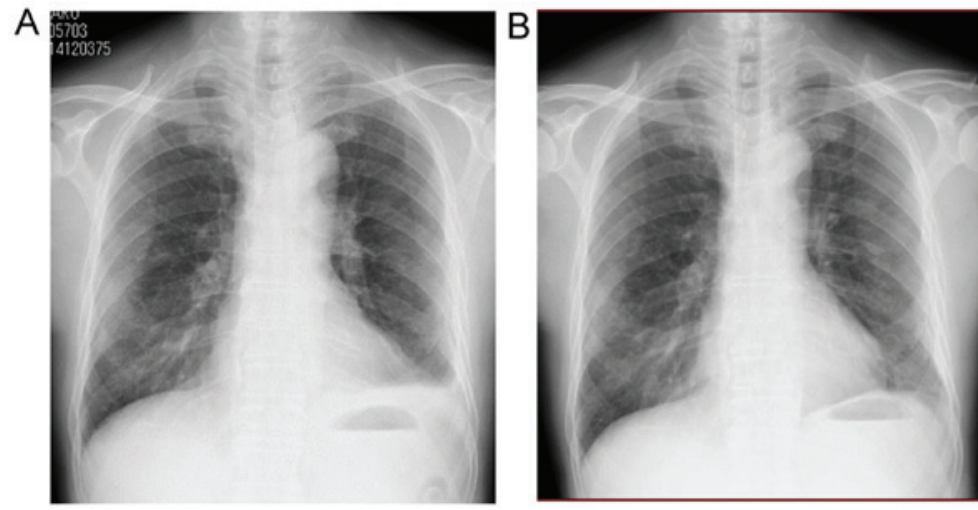

Figure 1. Chest radiography images. (A) Left pleural effusion is present. (B) Prior to the development of the left pleural effusion.
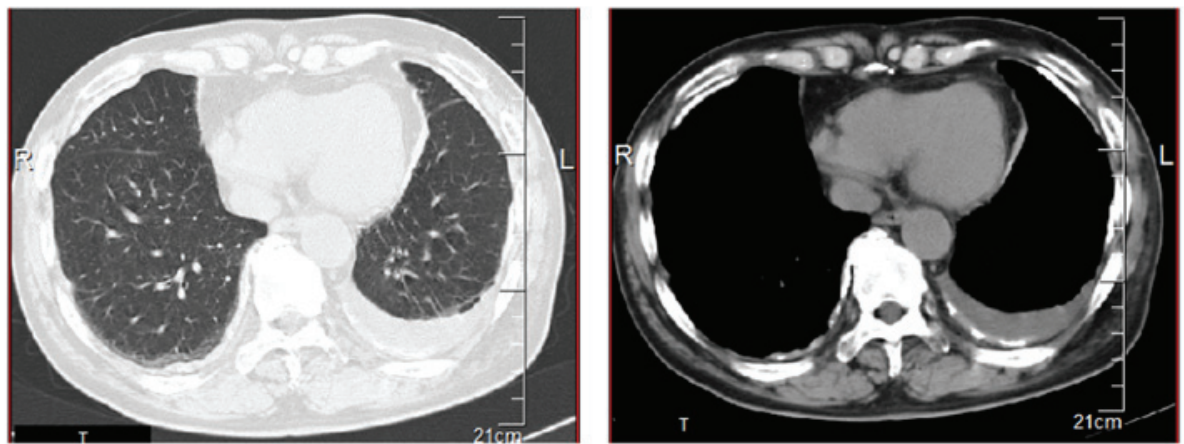

Figure 2. Chest computed tomography findings. Left pleural effusion is present. Thickening of the bilateral pleura and pleural plaques were also observed.

A

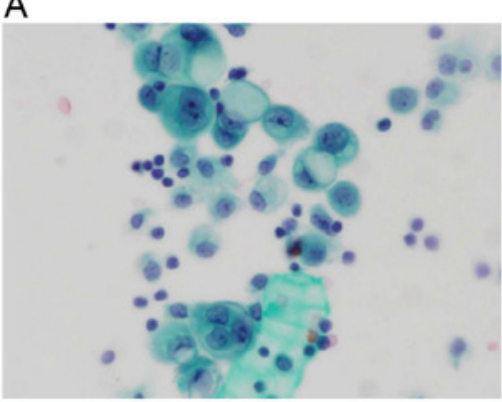

B

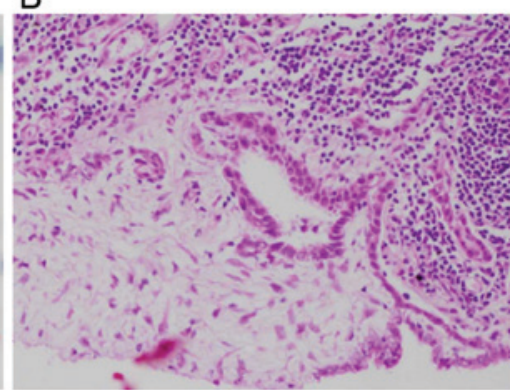

Figure 3. (A) Pleural fluid cytology confirmed malignant mesothelioma class V (Papanicolaou staining; magnification, x1,000). (B) Histological examination of the pleural biopsy revealed malignant pleural mesothelioma (hematoxylin and eosin staining; magnification, x200).

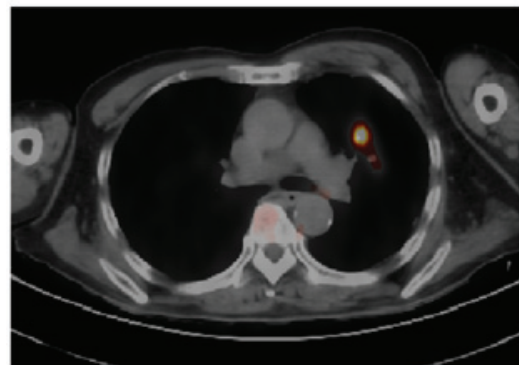

Figure 4. FDG positron emission tomography/computed tomography images. Nodular shadows measuring $20 \mathrm{~mm}$ with irregular margins and an FDG uptake with a maximum standardized uptake value of 5.48 are present in two locations in $\mathrm{S} 3$ of the left lung. The lesions were considered to represent a primary focus and a non-continuous additional tumor nodule in the same lung lobe; thus, clinical T3 was diagnosed. FDG, fluorodeoxyglucose. $\left(1,000 \mathrm{mg} / \mathrm{m}^{2}\right.$ on days 1,8 and 15 then every 28 days $)$. However, the pathological status of the patient worsened, and he eventually succumbed to lung cancer in November 2013.

\section{Discussion}

Malignant pleural mesothelioma (3) and lung cancer (4) are typical malignancies of the chest caused by asbestos exposure. In Japan, cases of malignant mesothelioma and asbestos-related lung cancer have been increasing due to the effects of asbestos that was extensively used in the past. The prognosis of malignant pleural mesothelioma is extremely dismal, with poor treatment outcomes. The efficacy of treatment is low, even when the disease is diagnosed at an early clinical stage. 

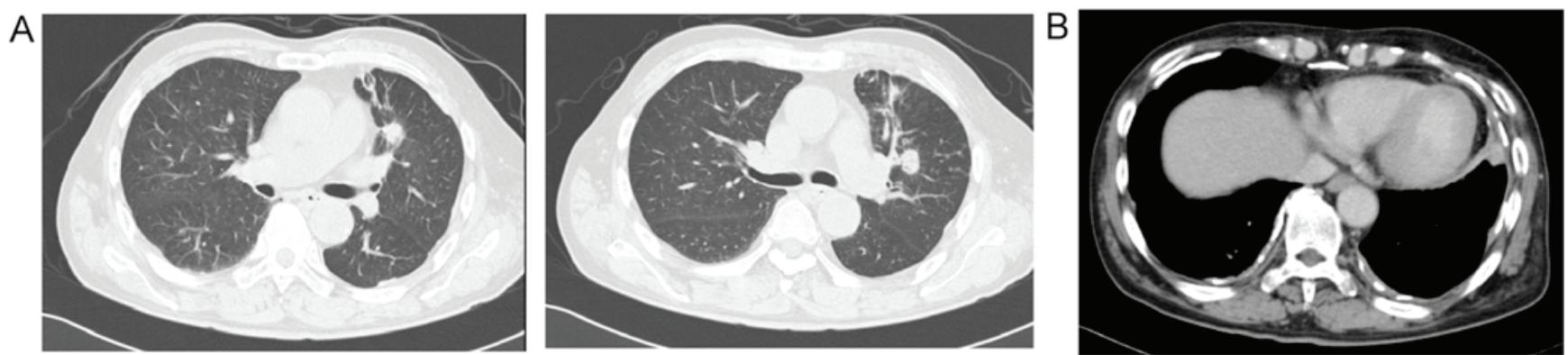

Figure 5. Chest contrast-enhanced computed tomography images. (A) A reticulate shadow in both lung fields is present, along with scattered nodular shadows with a centrilobular distribution, which were diagnosed as changes due to asbestosis. Two 20-mm nodular shadows with irregular margins were identified in S3 of the left lung. (B) Thickening of the bilateral pleura and pleural plaques are present, along with thickening of the left interlobular pleura. Contrast enhancement is evident in several locations, suggesting exacerbation of the mesothelioma.

A

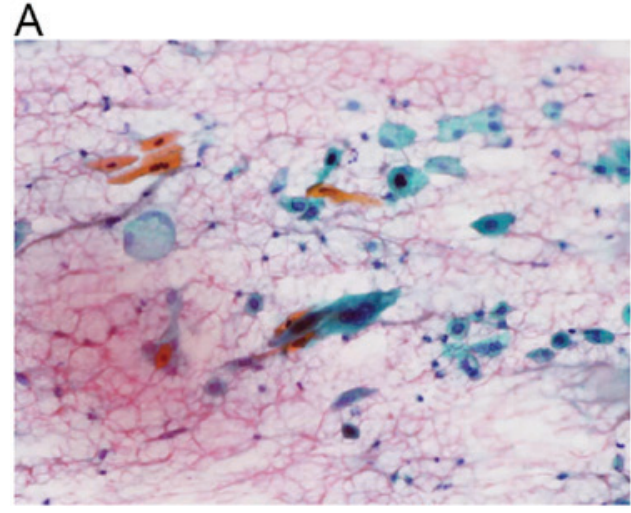

B

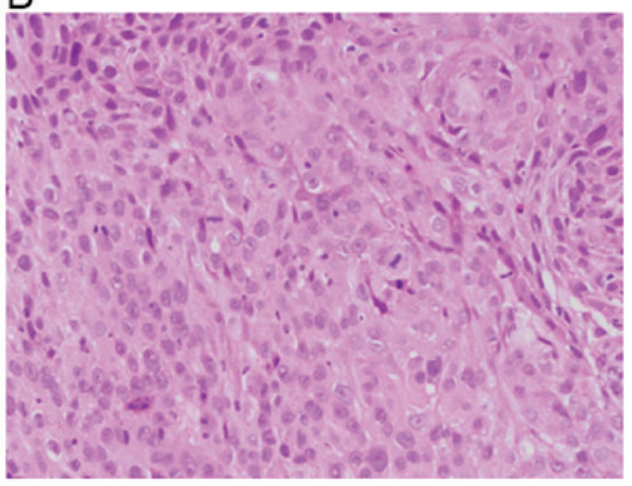

Figure 6. Bronchoscopy findings. (A) Brush cytology of the left S3 nodular shadows indicated squamous cell carcinoma, class V (Papanicolaou staining; magnification, $\mathrm{x} 400$ ). (B) Histological examination confirmed a diagnosis of squamous cell carcinoma (hematoxylin and eosin staining; magnification, $\mathrm{x} 400$ ).

A variety of multimodal treatment approaches, combining chemotherapy, surgery and radiotherapy, have been used. Trimodal therapy consisting of preoperative chemotherapy, EPP and postoperative hemithoracic radiotherapy, was reported to be effective in the treatment of malignant pleural mesothelioma, although high rates of surgical complications and perioperative mortality were observed (5). In contrast to the high incidence of surgical complications and perioperative mortality reported by Krug et al the MARS study (6) reported that EPP added no benefit to trimodal therapy, with other reports showing that pleurectomy/decortication $(\mathrm{P} / \mathrm{D})$ achieves better survival outcomes compared with EPP $(7,8)$, and indicating the possibility that treatment outcomes are improved by conserving the lung on the affected side. Recently, there has been a tendency toward using P/D reduction surgery in the treatment of early-stage cases of mesothelioma in which gross complete resection can be obtained.

To date, trimodal therapy has been implemented with total hemithoracic radiotherapy when EPP was the procedure used after preoperative chemotherapy. Although thoracic irradiation following P/D has been reported to have a high feasibility and efficacy in multimodal treatment for mesothelioma at an early stage (9), radiotherapy following lung dose constraints may make radiotherapy after $\mathrm{P} / \mathrm{D}$ of the affected lung challenging, and is currently contraindicated after surgery (10). Therefore, bimodal therapy (chemotherapy plus surgery) is typically used when $\mathrm{P} / \mathrm{D}$ is performed.
In the present case of double cancer, the patient developed lung SCC while receiving treatment for malignant pleural mesothelioma. Our findings suggest that the radical radiotherapy used for the treatment of the SCC may have also resulted in local control of the pre-existing malignant pleural mesothelioma, although only as a side effect (Fig. 7).

The present case confirms the value of radiotherapy in the treatment of malignant pleural mesothelioma, with only minor side effects, despite it being a radical and localized treatment for lung cancer. Since P/D is becoming the prevailing surgical procedure used in the treatment of early-stage cases of malignant pleural mesothelioma, a literature search was performed and it was considered whether radiotherapy could be added to treat the conserved lung after P/D.

Gupta et al implemented total hemithoracic radiotherapy of the affected side following $\mathrm{P} / \mathrm{D}$ in patients with malignant pleural mesothelioma (median dose, $42.5 \mathrm{~Gy}$; range, 7.2-67.8 Gy), but reported poor results, with a median survival of 12.5 months and a 2-year survival rate of $23 \%$ (11). They also reported being unable to increase the radiation dose to a level sufficient to destroy the tumor due to radiation-induced toxicity in the residual lung, and concluded that hemithoracic radiotherapy of the affected side following P/D was not an effective treatment option (11). However, since the emergence of intensity-modulated radiotherapy (IMRT), it has been demonstrated that radical irradiation is possible, even in the treatment of the conserved lung (12). Minatel et al 
A

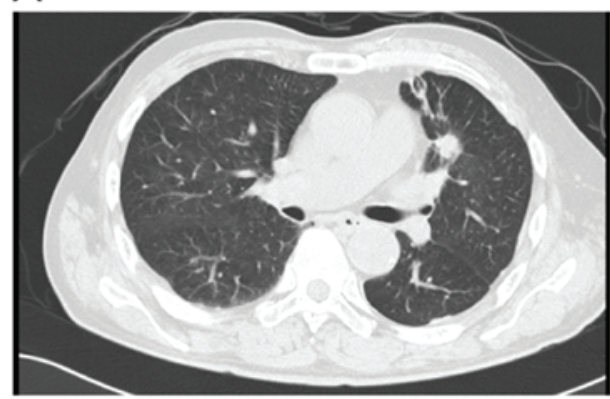

B

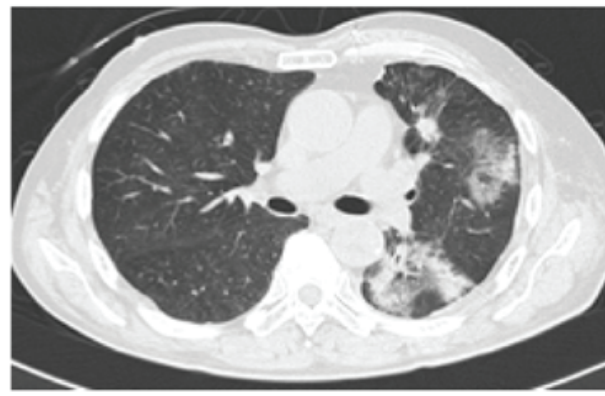

C

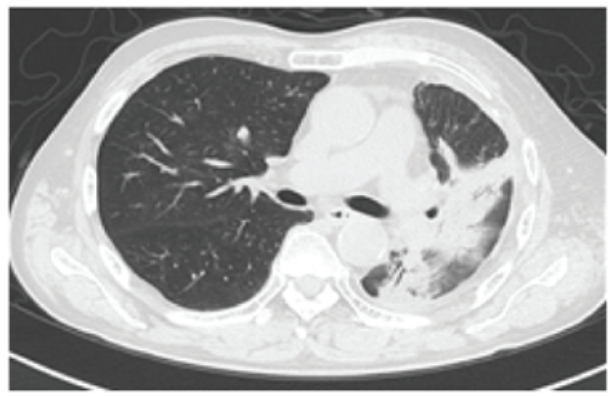

D

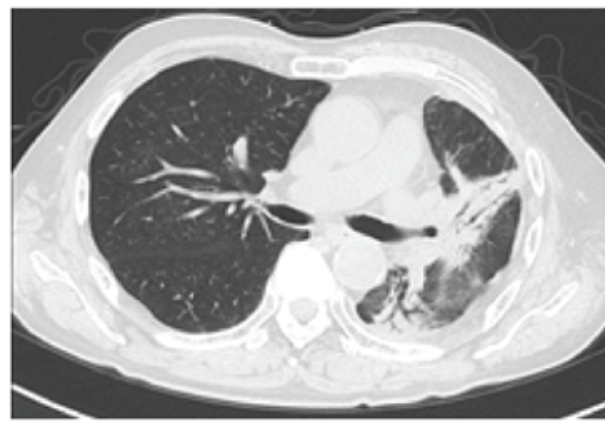

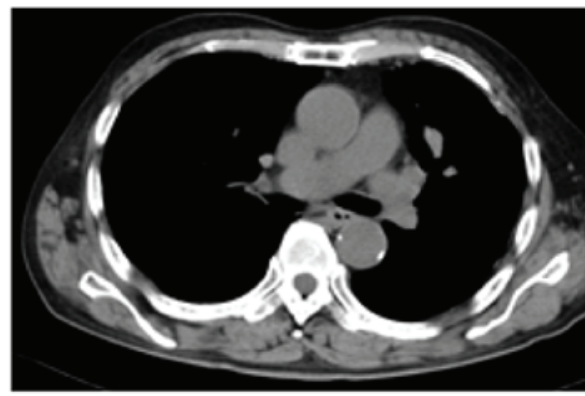
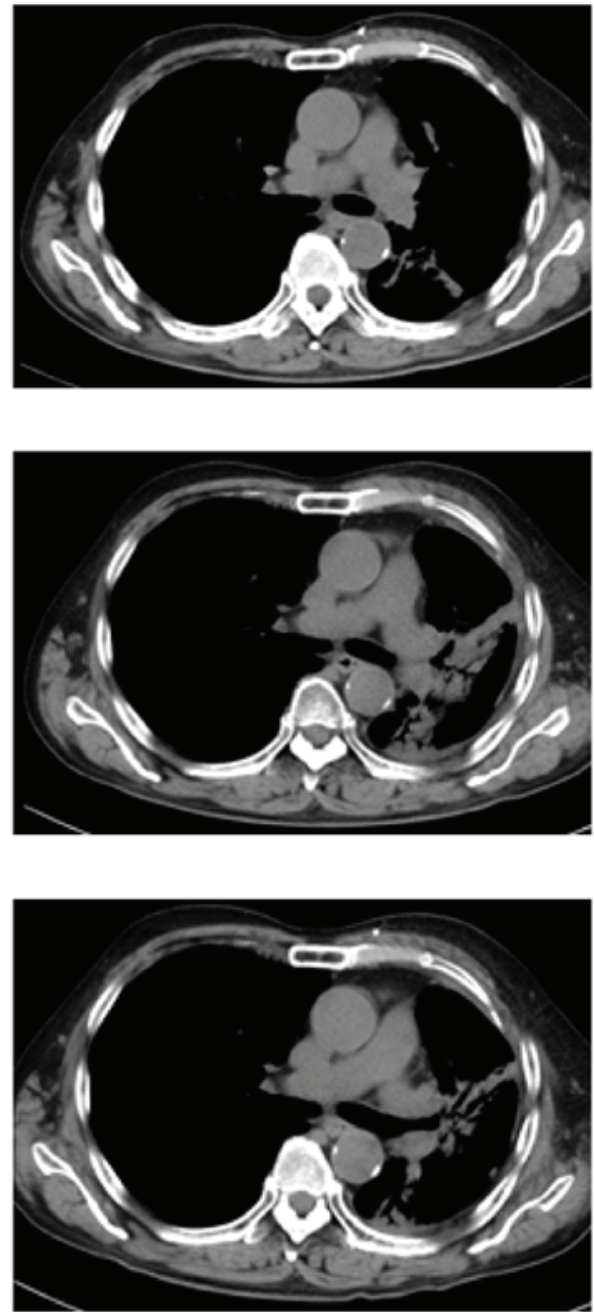

Figure 7. Progression of squamous cell lung cancer while under treatment for malignant pleural mesothelioma. (A) Prior to the start of chemoradiotherapy; (B) 3 months after the start of chemoradiotherapy; (C) 7 months after the start of chemoradiotherapy; and (D) 11 months after the start of chemoradiotherapy.

performed extended P/D in 35 of 69 patients with malignant pleural mesothelioma and partial pleurectomy in the remaining 34 patients. After implementing postoperative IMRT (50 Gy/25 fr) with a simultaneous boost of $60 \mathrm{~Gy}$ in 25 fractions (2.4 Gy per fraction) for residual disease, favorable 2-year survival rates of 65 and 58\%, respectively, were achieved in the two groups (13). They also reported that complications from IMRT did not cause treatment interruptions in any of the patients; the scheduled irradiation was completed in all patients, and complications were generally within a tolerable range (13).
In this report, the patient received radiotherapy of 60 Gy in 20 fractions (3 Gy per fraction). This dosing may result in a similar to slightly greater local effect on mesothelioma in terms of the biologically effective dose (BED), using the linear-quadratic model with an assumed $\alpha / \beta$ ratio of $10 \mathrm{~Gy}$ for the tumor $\left(\mathrm{BED}_{10}\right)$, since the $\mathrm{BED}_{10}$ is $78 \mathrm{~Gy}$ and $74.4 \mathrm{~Gy}$ for $60 \mathrm{~Gy} / 20 \mathrm{fr}$ and $60 \mathrm{~Gy} / 25 \mathrm{fr}$, respectively. Therefore, we suggest that radiotherapy at a dose $>60$ Gy may be used as local therapy in a conventional treatment schedule for mesothelioma (14).

Favorable outcomes have recently been obtained with IMRT following P/D. Moreover, an increasing number of 
reports, mainly from the USA and Europe, have indicated that this treatment is well-tolerated by the patients. Radiotherapy is not currently performed on the conserved lung after P/D in Japan; however, based on the present case and reports from outside Japan, there appears to be sufficient grounds to consider the suitability of this treatment modality.

In conclusion, the present case highlights the application of radiotherapy in the treatment of malignant mesothelioma. In addition, the potential of new multimodal treatments for mesothelioma and a comprehensive review of the literature are presented.

\section{Acknowledgements}

Not applicable.

\section{Funding}

No funding was received.

\section{Availability of data and materials}

All data generated or analyzed during this study are included in this published article.

\section{Authors' contributions}

YN, HD and NF contributed to the conception of the work and the acquisition, analysis and interpretation of data. YN analyzed and interpreted the patient data regarding the hematological disease. YK, EF and TM performed the histological examination of the lung and pleura. KK, KM and TM were responsible for drafting the manuscript and revising it critically for important intellectual content. TM was a major contributor to writing the manuscript. KK, TY and TK agree to be accountable for all aspects of the work in ensuring that questions related to the accuracy or integrity of any part of the work are appropriately investigated and resolved. All authors have read and approved the final manuscript.

\section{Ethics approval and consent to participate}

Not applicable.

\section{Patient consent for publication}

Appropriate written informed consent was obtained for the publication of this case report and the accompanying images.

\section{Competing interests}

The authors declare that they have no competing interests.

\section{References}

1. Allen TC and Moran C: Synchronous pulmonary carcinoma and pleural diffuse malignant mesothelioma. Arch Pathol Lab Med 130: 721-724, 2006.

2. Perrot M, Wu L, Wu M and Cho BCJ: Radiotherapy for the treatment of malignant pleural mesothelioma. Lancet Oncol 18 : e532-e542, 2017
3. Whitewell F and Rawcliffe RA: Diffuse malignant pleural mesothelioma and asbestos exposure. Thorax 26: 6-22, 1971 .

4. Doll R: Mortality from lung cancer in asbestos workers. Brit J Indust Med 12: 81-86, 1955.

5. Krug LM, Pass HI, Rusch VW, Kindler HL, Sugarbaker DJ, Rosenzweig KE, Flores R, Friedberg JS, Pisters K, Monberg M, et al: Multicenter phase II trial of neoadjuvant pemetrexed plus cisplatin followed by extrapleural pneumonectomy and radiation for malignant pleural mesothelioma. J Clin Oncol 27: 3007-3013, 2009.

6. Treasure T, Lang-Lazdunski L, Waller D, Bliss JM, Tan C, Entwisle J, Snee M, O'Brien M, Thomas G, Senan S, et al: Extra-pleural pneumonectomy versus no extra-pleural pneumonectomy for patients with malignant pleural mesothelioma: clinical outcomes of the Mesothelioma and Radical Surgery (MARS) randomized feasibility study. Lancet Oncol 12: 763-772, 2011.

7. Flores RM, Pass HI, Seshan VE, Dycoco J, Zakowski M, Carbone M, Bains MS and Rusch VW: Extrapleural pneumonectomy versus pleurectomy/decortications in the surgical management of malignant pleural mesothelioma: Results in 663 patients. J Thoracic Cardiovasc Surg 135: 620-626, 2008.

8. Lang-Lazdunski L, Bille A, Lal R, Cane P, McLean E, Landau D, Steele J and Spicer J: Pleurectomy/decortication is superior to extrapleural pneumonectomy in the multimodality management of patients with malignant pleural mesothelioma. J Thorac Oncol 7: 737-743, 2012.

9. Shaikh F, Zauderer MG, von Reibnitz D, Wu AJ, Yorke ED, Foster A, Shi W, Zhang Z, Adusumilli PS, Rosenzweig KE, et al: Improved outcomes with modern lung-sparing trimodality therapy in patients with malignant pleural mesothelioma. J Thorac Oncol 12: 993-1000, 2017.

10. Scherpereel A, Astoul P, Baas P, Berghmans T, Clayson H, de Vuyst P, Dienemann H, Galateau-Salle F, Hennequin C, Hillerdal G, et al: Guidelines of the european respiratory society and the european society of thoracic surgeons for the management of malignant pleural mesothelioma. Eur Respir J 35: 479-495, 2010 .

11. Gupta V, Mychalczak B, Krug L, Flores R, Bains M, Rusch VW and Rosenzweig KE: Hemithoracic radiation therapy after pleurectomy/decortication for malignant pleural mesothelioma. Int J Radiat Oncol Biol Phys 63: 1045-1052, 2005.

12. Rosenzweig KE, Zauderer MG, Laser B, Krug LM, Yorke E, Sima CS, Rimner A, Flores R and Rusch V: Pleural intensity-modulated radiotherapy for malignant pleural mesothelioma. Int J Radiat Oncol Biol Phys 83: 1278-1283, 2012.

13. Minatel E, Trovo M, Bearz A, Di Maso M, Baresic T, Drigo A, Barresi L, Furlan C, Del Conte A, Bruschi G, et al: Radical radiation therapy after lung sparing surgery for malignant pleural mesothelioma: Survival, pattern of failure and prognostic factors. Int J Radiation Oncol Biol Phys 93: 606-613, 2015.

14. Hall EJ and Giaccia AJ: Radiobiology for the radiologist. 7th ed. Lippincott Williams \& Wilkins, Philadelphia, 2011. 